Florian Kunze

\title{
Dealing with the Demographic Change in Companies - Potential Impacts and Intervention Strategies for an Aging Workforce
}

Betreuer: Prof. Dr. Heike Bruch, Universität St. Gallen, Prof. Dr. Sven Voelpel, Jacobs Universität Bremen

\section{Ausgangslage}

Der demographische Wandel ist eine der zentralen Herausforderungen für Wirtschaft und Gesellschaft. Insbesondere für Unternehmen ist es relevant, die Konsequenzen der Alterung und veränderten Altersstruktur ihrer Belegschaften zu kennen und Inter ventionsmöglichkeiten zum Erhalt ihrer Wettbewerbsfähigkeit zu besitzen. Um die Auswirkungen des demographischen Wandels in und für Unternehmen möglichst um fassend zu verstehen, bietet sich eine Strukturierung nach drei unterschiedlichen Be trachtungsebenen an: (1) Der Ebene des individuellen Beschäftigten, (2) der Ebene von Teams und (3) der Ebene des Gesamtunternehmens. Trotz zunehmender For schung in diesem Bereich gibt es auf allen drei Betrachtungsebenen noch eine Vielzahl blinder Flecken, die einer fundierten empirischen Untersuchung bedürfen. Die vorlie gende Arbeit greift auf jeder Analyseebene eine offene Fragestellung auf und unter sucht in drei empirischen Studien (1) den Widerstand gegen Wandel und Leistungsfä higkeit älterer Mitarbeiter, (2) die Auswirkungen von Altersvielfalt in Teams und (3) die Konsequenzen von Altersvielfalt im Gesamtunternebmen.

\section{Theoretische Grundlagen}

Die drei Studien der Dissertation bedienen sich unterschiedlicher theoretischer Litera turstränge aus verschiedenen Bereichen der Personal und Organisationsforschung. Die wichtigsten theoretischen Argumente für jede Studie sollen im Folgenden kurz skizziert werden.

Die Studie auf individueller Ebene greift die Fragestellung auf, ob ältere Arbeitneh mer, entgegen der gängigen Altersvorurteile, weniger Widerstand gegen organisationa len Wandel aufweisen als ihre jüngeren Kollegen. Dies könnte zum einen damit erklärt werden, dass ältere Mitarbeiter nach den Ergebnissen der neuropsychologischen For schung bessere Emotionsregulationskompetenzen besitzen sollten, was ihnen im Um gang mit emotional herausfordernden Ereignissen, wie zum Beispiel Wandelprozes sen, zu gute kommen sollte. Des Weiteren ist auch davon auszugehen, dass ältere Mit arbeiter ein höheres Selbstvertrauen besitzen, schwierige Aufgaben am Arbeitsplatz er folgreich zu bewältigen, da sie in ihrer Erwerbsbiographie wahrscheinlich schon wie derholt mit Herausforderungen konfrontiert waren. Dieser geringere Widerstand ge gen Wandel sollte sich auch wiederum positiv auf die individuelle Leistungsfähigkeit auswirken.

Die Studie auf der Teamebene greift die Diskussion der Diversitätsforschung auf, ob sich Altersdiversität positiv oder negativ auf Gruppenprozesse auswirkt. Entspre chend der sozialen Identitätstheorie wird vermutet, dass sich unterschiedliche Alters 
gruppen zunächst voneinander abgrenzen, insbesondere wenn Alter gleichzeitig mit anderen Diversitätsmerkmalen in Form von sogenannten „Faultlines“ auftritt. Die ne gativen Auswirkungen der Gruppenleistungen können jedoch überwunden werden, wenn durch ein transformationales Führungsklima eine gemeinsame Gruppenidentität geschaffen wird, die die Altersidentitäten überlagert.

Schließlich bedient sich die Studie auf Gesamtunternebmensebene ebenfalls Argumen ten aus der Diversitätsforschung. Analog zu der Studie auf der Teamebene ist davon auszugehen, dass Altersvielfalt auch über Team und Abteilungsgrenzen hinweg nega tive Konsequenzen haben kann. Alter dürfte auch im gesamten Unternehmen ein Fak tor sein, über den sich Mitarbeiter differenzieren und zu Untergruppen abgrenzen, was zuerst zu einer Zunahme von Altersdiskriminierung im Unternehmen führen kann. Wenn das Altersdiskriminierungsklima hoch ist, kann dies in Konsequenz zu ei nem Rückgang des kollektiven Commitments der Mitarbeiter führen, was sich wiede rum negativ auf die Gesamtunternehmensleistung auswirken kann.

\section{Zentrale Ergebnisse der Arbeit}

Studie 1 - Individuelle Ebene:

Alter, Widerstand gegen Wandel und individuelle Leistungsfähigkeit

Zur Überprüfung der Fragestellungen auf der individuellen Ebene wurden 3776 Mit arbeiter in 93 deutschen Firmen zu ihrem Widerstand gegen Organisationalen Wandel und ihrem Alter befragt und statistische Auswertungen mit Hilfe von Strukturglei chungsmodellen vorgenommen. Überraschenderweise gab es, entgegen dem gängigen Vorurteil, als Ergebnis einen leicht negativen, signifikanten Zusammenhang zwischen Alter und Widerstand gegen Wandel. Dieser Zusammenhang wurde darauf, im zwei ten Schritt, noch genauer unter Berücksichtigung der Betriebszugehörigkeit und der Tätigkeit der Mitarbeiter (Angestellte oder Arbeiter) untersucht. Beide Faktoren erwie sen sich als wichtige Rahmenbedingungen. So konnte gezeigt werden, dass ältere Mit arbeiter, die nur für kurze Zeit im Unternehmen gearbeitet hatten, einen sehr starken negativen Zusammenhang zwischen Alter und Widerstand gegen Wandel aufzeigten, wohingegen für diejenigen Mitarbeiter mit einer langen Betriebszugehörigkeit der Zu sammenhang leicht positiv war. Ebenso ergab sich für Angestellte ein weitaus stärke rer negativer Zusammenhang als für Arbeiter. Als dritter Schritt untersuchte die Studie auch noch die individuellen Leistungsimplikationen des Widerstands gegen Wandel. In Einklang mit den theoretischen Annahmen konnte gezeigt werden, dass Mitarbeiter, die unflexibler sind, auch geringere Leistung in Form der Einreichung von Verbesse rungsvorschlägen und der individuellen Zielerreichung erbringen. Außerdem hatten Mitarbeiter mit einem höheren Widerstand gegen Wandel auch mehr Fehlzeiten auf grund gesundheitlicher Probleme. Dadurch konnte eine Kausalkette von Alter über Widerstand gegen organisationalen Wandel hin zu individueller Leistungsfähigkeit aufgezeigt werden.

Studie 2 - Team Ebene:

Altersdiversität, Teamprozesse und der Einfluss von Führungsverbalten

In der zweiten Studie der Dissertation wurden die theoretischen Annahmen zu den Zusammenhängen auf Gruppenebene in einer Stichprobe von 72 Arbeitsgruppen ei 
nes internationalen Maschinenbaukonzerns getestet, indem quantitative Befragungen von allen Teammitgliedern durchgeführt wurden. Entsprechend den theoretischen Voraussagen hatten altersbasierte Verwerfungslinien (Subgruppen, die sich aufgrund von Alter in Kombination mit Geschlecht und Betriebszugehörigkeit bilden) einen negativen Effekt auf die produktive Teamenergie. Das heißt, dass in solchen vielfälti gen Arbeitsgruppen zunächst wirklich die negativen Auswirkungen überwiegen und verhindern, dass die gemeinsamen geistigen, emotionalen und verhaltensorientierten Potenziale aktiviert werden und im Hinblick auf gemeinsame Ergebnisse umgesetzt werden. Wenn es innerhalb dieser diversen Teams ein hohes transformationales Ver halten der Führungskraft gibt, werden die negativen Effekte ins Positive umgekehrt. Einer Führungskraft, die gemeinsame Ziele vorgibt und vorlebt, charismatisch auftritt und auch die individuellen Kompetenzen der einzelnen Mitarbeiter entwickelt, scheint es also wirklich zu gelingen, die Potenziale von Vielfalt in Teams zu aktivieren.

\section{Studie 3 - Unternebmensebene:}

\section{Altersdiversität, Altersdiskriminierung und Unternebmensleistung}

Die Zusammenhänge zwischen Altersdiversität, Altersdiskriminierung und Unter nehmensleistung wurden in der dritten Studie in einer Stichprobe von 128 klein und mittelständischen Unternehmen mit mehr als 18.000 Mitarbeitern untersucht. Zur Vermeidung einer Verzerrung durch eine „same source bias“ wurden 4 unterschiedli che Datenquellen benutzt (2 unterschiedliche Mitarbeiterfragebögen, Befragung der Geschäftsführung und Befragung der Personalverantwortlichen). Innerhalb dieser Stichprobe konnten die Hypothesen zur Auswirkung des demographischen Wandels auf Unternehmensebene mit Hilfe von Strukturgleichungsmodellen bestätigt werden. Altersvielfalt zeigte einen klar positiven Zusammenhang mit einem Altersdiskriminie rungsklima in den Unternehmen. Dieses wirkte sich wiederum negativ auf das kollek tive Commitment der Mitarbeiter und letztendlich auch auf die Unternehmensgesamt leistung aus. Insofern konnte der demographische Wandel klar auch als Herausforde rung für die Gesamtorganisation identifiziert werden.

\section{Literatur}

Kunze, F., Boehm, S., \& Bruch, H. (2011). Age Diversity, Age Discrimination, and Performance Conse quences a Cross Organizational Study. Journal of Organizational Behavior, 32(2), 264290.

Kunze, F., \& Bruch, H. (2010). Age Based Faultlines and Perceived Productive Energy: The Moderation of Transformational Leadership. Small Group Research, 41(5), 593620.

Kunze, F., Boehm, S., \& Bruch, H. (2010). Age, resistance to change, and job performance: Testing for a common stereotype. In L. Toombs (Ed.), Best Paper Proceedings of the Seventieth Annual Meeting of the Academy of Management, Montreal, QB. 